\title{
Fundus Examination to Guide Anticoagulation Therapy in Suspected COVID-19 in a Critical Care Unit
}

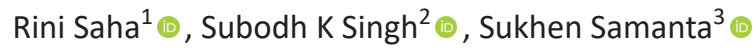 \\ Keywords: Coronavirus disease 2019, D-Dimer, Retinal vein thrombosis. \\ Indian Journal of Critical Care Medicine (2021): 10.5005/jp-journals-10071-23865
}

\section{INTRODUCTION}

Coagulation derangements like elevated D-dimer levels, prothrombin time and partial thromboplastin time prolongation, and increase in fibrin degradation products are common in patients with severe acute respiratory syndrome coronavirus 2 (SARS-CoV-2) infection. Several studies have looked into retinal changes in coronavirus disease 2019 (COVID-19) patients. ${ }^{1,2}$ One of the ocular manifestations of COVID-19 is retinal vein thrombosis (RVT). We report a case of bilateral impending RVT in a COVID-19suspected patient with raised D-dimer emphasizing the role of ocular examination in COVID-19 patient management.

\section{Case Report}

A 19-year-old COVID-19-suspected patient was admitted in a high dependency ward on nasal prong oxygen therapy with complaints of fever, cough, difficulty breathing, and anosmia for last 10 days. The patient was referred to the ophthalmologist for blurred vision in her right eye for the last 4 days. Due to the current ongoing COVID-19 pandemic and high clinical suspicion, a real-time reverse transcription-polymerase chain reaction (RT-PCR) for SARS-CoV-2 from a nasopharyngeal swab was performed but was negative. An IgG/lgM Rapid Test was also negative for SARS-CoV-2. The D-dimer was high (3430 $\mathrm{ng} / \mathrm{mL}$ ) and so was the C-reactive protein (98.8 $\mathrm{mg} / \mathrm{L})$. Other hypercoagulability markers were all within the normal limit. Serum lactate dehydrogenase level was elevated with mild lymphopenia. Rest laboratory evaluations were normal. High-resolution computed tomography of the chest showed mild bilateral lung parenchyma involvement. There was no evidence of systemic venous thrombosis. Echocardiography and bilateral leg Doppler reports showed no deep venous thrombosis. Ophthalmic examination at presentation showed best-corrected visual acuity of 20/30 in the right eye (OD) and 20/20 in the left eye (OS). There was no afferent pupillary defect. Slit lamp examination showed normal anterior segment findings, including intraocular pressure OU. Fundus examination revealed dilated retinal veins OU. There were few epiretinal hemorrhages in OD, including one at the fovea (Figs 1 and 2). A diagnosis of bilateral impending RVT in suspected SARS-CoV-2 was made. Due to high D-dimer, the patient was on a prophylactic dose of low molecular weight heparin (LMWH). Due to evidence of systemic (retinal) venous thrombosis, the treating physician decided to increase the dose of $\mathrm{LMWH}$ from prophylactic to therapeutic. The patient was reevaluated at monthly followups to complete resolution of ocular signs and symptoms and normalization of her serum D-dimer levels.

\author{
1,2Department of Ophthalmology, IRIS Super Speciality Eye Hospital, \\ Ranchi, Jharkhand, India \\ ${ }^{3}$ Department of Critical Care, Orchid Medical Centre, Ranchi, \\ Jharkhand, India \\ Corresponding Author: Sukhen Samanta, Department of Critical \\ Care, Orchid Medical Centre, Ranchi, Jharkhand, India, Phone: \\ +919142928038, e-mail: dr.sukhensamanta@gmail.com \\ How to cite this article: Saha R, Singh SK, Samanta S. Fundus \\ Examination to Guide Anticoagulation Therapy in Suspected COVID-19 \\ in a Critical Care Unit. Indian J Crit Care Med 2021;25(6):737-738. \\ Source of support: Nil \\ Conflict of interest: None
}

\section{Discussion}

Proposed mechanisms for multiorgan dysfunction syndrome in COVID-19 are multifactorial, including cytokine storm and a hypercoagulable state, with micro- and macrocirculatory thrombosis. Elevated D-dimer (above $1 \mu \mathrm{g} / \mathrm{mL}$ ) is a strong and independent risk factor for death in this population. This has led to an interest in the potential uses of anticoagulation in COVID-19 specifically heparin. It has been recommended that all patients with COVID-19 should undergo coagulation studies, in particular: D-dimer, prothrombin time, and platelet count. If the coagulation profile is deranged, prophylactic doses of anticoagulation are advisable. ${ }^{3}$ Therapeutic anticoagulation should be strongly considered in patients demonstrating signs of microthrombiinduced organ dysfunction or with documented or strongly suspected macrothromboembolism. ${ }^{4}$ The arteries and veins of the retina represent a window to the vascular system showing changes in many systemic diseases. ${ }^{5}$ Our patient had raised D-dimer without evidence of systemic micro- and macrocirculatory thrombosis. Ocular examination revealed impending RVT, and hence, the dose of systemic anticoagulation was changed from prophylactic to therapeutic. RVT can be vision threatening, and timely therapeutic anticoagulation therapy prevented a blinding condition in our patient.

\section{ConClusion}

Eye examination can be an important aspect of evaluation in COVID-19-suspected patients providing insight into optimal anticoagulation management in this subset of patients. The patient's written consent was received for writing this case report. 


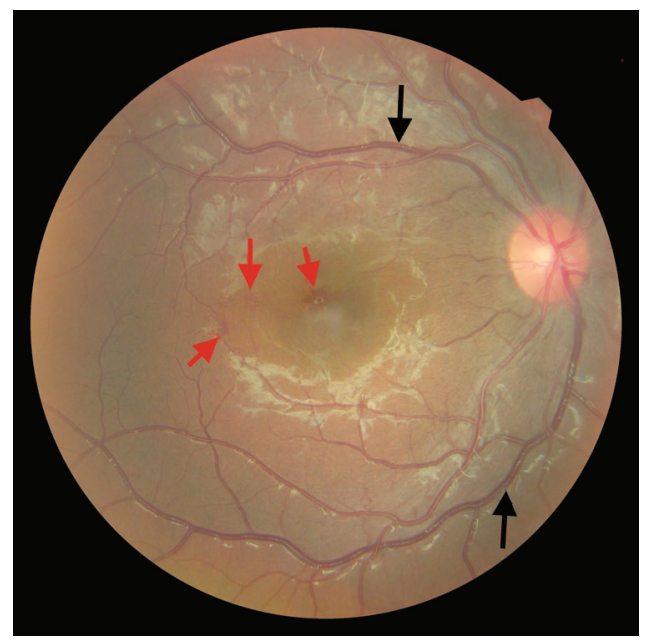

Fig. 1: Fundus picture of the right eye with arrows

\section{OrCID}

Rini Saha ำ https://orcid.org/0000-0001-8516-4634

Subodh K Singh (ㄱ) https://orcid.org/0000-0001-6662-1123

Sukhen Samanta (i) https://orcid.org/0000-0002-3041-9529

\section{References}

1. Bertoli F, Veritti D, Danese C, Samassa F, Sarao V, Rassu N, et al. Ocular findings in COVID-19 patients: a review of direct manifestations and indirect effects on the eye. J Ophthalmol 2020;2020:4827304. DOI: 10.1155/2020/4827304.

2. Pirraglia MP, Ceccarelli G, Cerini A, Visioli G, d'Ettorre G, Mastroianni CM, et al. Retinal involvement and ocular findings in

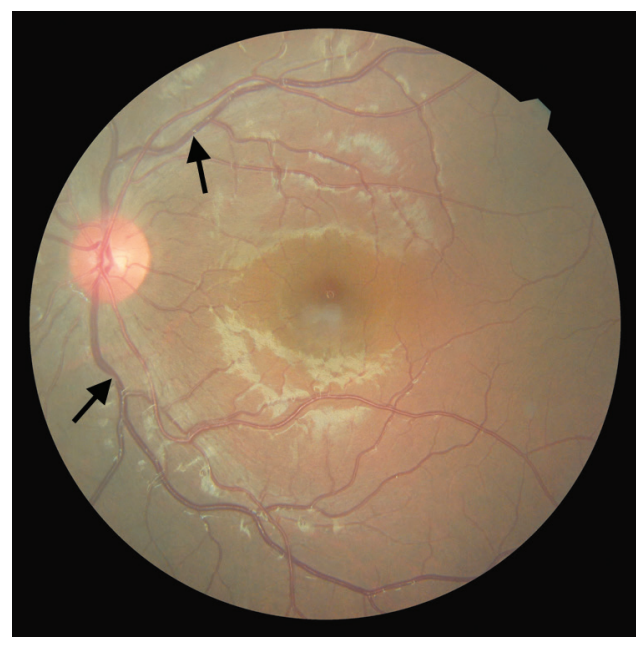

Fig. 2: Fundus picture of left eye with arrows

COVID-19 pneumonia patients. Sci Rep 2020;10(1):17419. DOI: 10.1038/ s41598-020-74446-6.

3. Tang N, Bai H, Chen X, Gong J, Li D, Sun Z. Anticoagulant treatment is associated with decreased mortality in severe coronavirus disease 2019 patients with coagulopathy. J Thromb Haemost 2020;18(5):10941099. DOI: 10.1111/jth.14817.

4. Thachil J, Tang N, Gando S, Falanga A, Cattaneo M, Levi M, et al. ISTH interim guidance on recognition and management of coagulopathy in COVID-19. J Thromb Haemost 2020;18(5):1023-1026. DOI: 10.1111/ jth.14810.

5. Invernizzi A, Torred A, Parrullia S, Zicarellia F, Schiumab M, Colombod V, et al. Retinal findings in patients with COVID-19: results from the SERPICO-19 study. EClinicalMedicine 2020. DOI: 10.1016/j.eclinm.2020.100550. 